\section{Kidney \\ Blood Pressure Research}

\title{
Protective Effect of Znt7 on High Glucose- Induced Epithelial-to-Mesenchymal Transition in Renal Tubular Epithelial Cells
}

\author{
Xiuli Zhanga,b,c Xu Lian ${ }^{\mathrm{a}} \quad$ Dan Liang $^{\mathrm{e}}$ Lianzhi Zhang ${ }^{\mathrm{a}} \quad$ Shengquan Liu ${ }^{\mathrm{e}}$ \\ Lina Yang ${ }^{f}$ Zhi-Hong Chia Harvest F. Gug
}

aDepartment of Pathophysiology, China Medical University, Shenyang, Liaoning, bepartment of Nephrology, Benxi Center Hospital, Benxi, Liaoning, 'Key Laboratory of Medical Cell Biology, Chinese Ministry of Education, China Medical University, Shenyang, Liaoning, dDepartment of Endocrinology, The Affiliated Hospital of Mudanjiang Medical University, Mudanjiang, Heilongjiang, ${ }^{\text {TTroops of }}$ 95988 Unit, Changchun, Jilin, 'Department of Geriatrics, The First Affiliated Hospital of China Medical University, Shenyang, Liaoning, China, ${ }^{D}$ Department of Clinical Science, Intervention and Technology, Karolinska University Hospital, Karolinska Institutet, Stockholm, Sweden

\section{Key Words}

Epithelial-to-mesenchymal transition - High glucose - Renal tubular epithelial cells - SiRNA • The MAPK/ERK pathway • The TGF- $\beta /$ Smad pathway $\cdot$ Zinc transporter 7

\begin{abstract}
Background/Aims: Evidence from our and other groups has demonstrated that zinc transporter 7 in SLC30 family (ZnT7) inhibited epithelial-to-mesenchymal transition (EMT) and apoptosis in rat peritoneal mesothelial cells (RPMCs) under high glucose (HG) concentration. In the present study, we investigated the effect of ZnT7 on EMT of renal tubular epithelial cells (RTECs) in an in vitro model of diabetic nephropathy (DN). Methods: A dual-fluorescent staining protocol was used for detection of ZnT7 in a normal rat kidney tubular epithelial cell line (NRK-52E cells). EMT was induced with HG (30 mM). NRK-52E cells were transfected with plasmids codifying for hZnT7-EGFP and interfering RNA for determination of the effect of ZnT7 over-expression and silencing, respectively. Expression of ZnT7, activation of the MAPK/ ERK and TGF- $\beta /$ Smad pathways were analyzed with by means of Western blot. Results: ZnT7 was localized in the perinuclear region and Golgi apparatus. In HG-induced EMT of NRK-52E cells, ZnT7 was up-regulated. Over-expression of ZnT7 led to inhibition of HG-induced EMT, while knock-down of ZnT7 increased EMT. Furthermore, knock-down of ZnT7 and increased HG-induced EMT was accompanied by activation of the MAPK/ERK and TGF- $\beta / S m a d$ pathways. Conclusion: The present study provides evidence that ZnT7 has a protective effect over EMT
\end{abstract}




\section{Kidney Blood Pressure Research}

Kidney Blood Press Res 2018;43:500-512

\begin{tabular}{l|l}
\hline DOI: 10.1159/000488697 & (C) 2018 The Author(s). Published by S. Karger AG, Base
\end{tabular}

Published onlıne: 6 April, 2018

www.karger.com/kb

of RTECs in DN and suggests that the inhibition of HG-induced EMT may be achieved through the MAPK/ERK and TGF- $\beta /$ Smad pathways. Thereby, ZnT7 could be a potential target for translation medicine and prevention program in $\mathrm{DN}$.

\section{Introduction}

Zinc $(\mathrm{Zn})$ is an essential element in nucleic acid metabolism, cell replication, tissue repair and growth, while zinc homeostasis in the body is mainly regulated by zinc transporter systems, including zinc transporters of SLC30 family [1, 2]. In this family, ZnT7 is the $7^{\text {th }}$ member and expressed in brain, heart, lung, liver, kidneys, spleen, small intestine and peritoneum $[3,4]$. Moreover, ZnT7 is presented in the face of the Golgi apparatus and functionally transports cytoplasmic zinc into the Golgi network $[4,5]$. The trans-Golgi network (TGN) is part of the secretory pathway of eukaryotic cells which is distinct from the Golgi stack and important in the later stages of protein secretion. Trans-Golgi network 38 (TGN38) is an integral membrane protein and predominantly localized to the TGN in NRK cells [6]. Interestingly, a few reports have implicated that ZnT7 may be involved in the regulation of insulin and glucose metabolism. In 2010, Huang L et al. reported that ZnT7 was co-expressed with insulin in the pancreatic islets [7]. Two years later, her group demonstrated that insulin secretion was decreased while insulin resistance was increased in ZnT7 KO mice, which resulted in glucose intolerance [8]. Later on, we analyzed all ZnT members in SLC30 family and observed for the first time that ZnT7, under the condition of high glucose (HG), inhibited the epithelial-to-mesenchymal transition (EMT) in rat peritoneal mesothelial cells (RPMC) [9]. We further demonstrated that ZnT7 might inhibit HG-induced apoptosis by activation of the PI3K/Akt pathway [10]. Very recently, Tuncay et al. provided evidence that ZnT7 upregulation was observed in hyperglycemia, and alteration of ZnT7 activity could be involved in the pathogenesis of cardiac dysfunction in diabetes [11].

Diabetes is characterized by high plasma glucose levels resulting from defective insulin secretion, insulin resistance or both [12]. The patients with diabetes often develop diabetic nephropathy (DN), which is a micro-vascular complication and causes the progressive renal fibrosis, eventually reducing functional renal mass [13]. In DN, the fibrosis develops in the glomerulus and tubulointerstitium of kidneys. In general, the renal fibrosis in DN is considered as irreversible $[14,15]$ although the pathogenesis of DN is not fully understood. Recent studies have demonstrated that HG could induce the complex progress of proinflammatory and profibrotic stimuli EMT of tubular epithelial cells in kidneys $[16,17]$. Furthermore, the MAPK/ERK and TGF- $\beta$ /Smad signaling pathways have been reported to play a vital role in the EMT $[18,19]$. HG could up-regulate the expression of TGF- $\beta 1$, which is a strong inducer of EMT in the renal tubular epithelial cells [20]. Taking together the information from the studies of ZnT7 described above, we have a hypothesis that ZnT7 may have the protective effect on EMT of RTEC, which acts as an important mechanism in DN.

In the present study, we first detected the localization of ZnT7 in a normal rat kidney tubular epithelial cell line (NRK-52E cells). We then analyzed ZnT7 activities in the cells under HG conditions. We further investigated the possible role of ZnT7 in the TGF- $\beta /$ Smad and MAPK/ERK pathways. The aims of this study were to test our hypothesis and to accumulate the information for the application of ZnT7 as a potential target for translation medicine and prevention program in $\mathrm{DN}$.

\section{Materials and Methods}

Cell culture

Normal rat kidney tubular epithelial cell line (NRK-52E) was obtained from the American Type Culture Collection (Rockville, MD, USA) and cultured in Dulbecco's modified Eagle's medium (DMEM; low glucose; 


\section{Kidney \\ Blood Pressure Research}

Kidney Blood Press Res 2018;43:500-512

\begin{tabular}{l|l}
\hline DOI: $10.1159 / 000488697$ & (C) 2018 The Author(s). Published by S. Karger AG, Basel
\end{tabular}

Published onlıne: 6 April, 2018 www.karger.com/kbr

Gibco Life Technologies, Grand Island, NY, USA) containing $10 \%$ fetal bovine serum, $4 \mathrm{mM} \mathrm{L-glutamine,} \mathrm{and} \mathrm{1 \%} \mathrm{penicillin/}$ streptomycin. The cells were cultured at a density of $5 \times 10^{3}$ cells/well in 6-well culture plates. Near confluent NRK-52E cells were then transferred to serum free DMEM medium for overnight starvation prior to each experiment.

\section{Assessment of cell viability}

Cell viability was measured by quantitative colorimetric assay with $\quad 3$-(4, 5-dimethylthiazol-2-yl)-2, 5-diphenyltetrazolium bromide (MTT) in 96-well plates (Fig. 1). Briefly, at the indicated time after treatment, $10 \mu \mathrm{l}$ MTT (final concentration, $500 \mu \mathrm{g} / \mathrm{mL}$ ) was added

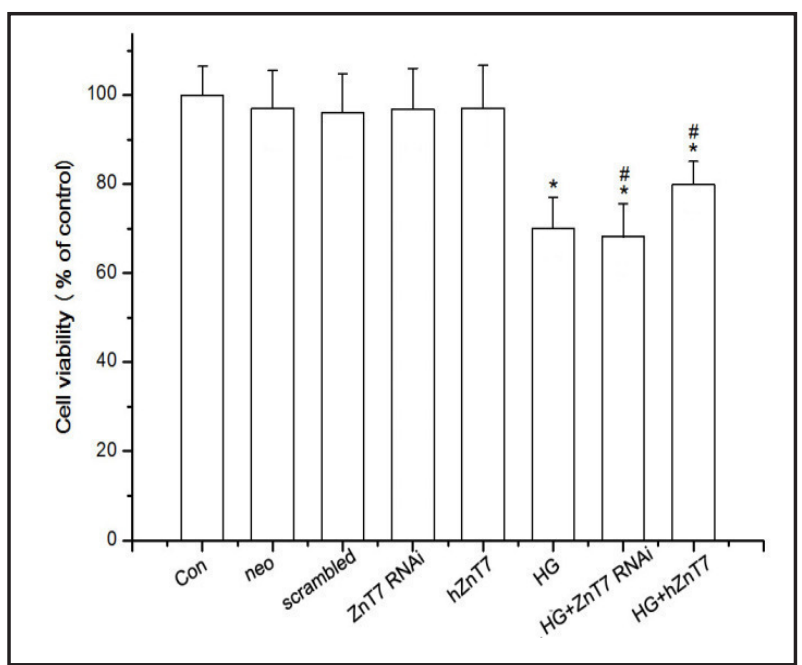

Fig. 1. MTT measurement of the cell viabilities. to the medium and incubated at $37^{\circ} \mathrm{C}$ for 3 $\mathrm{h}$. The MTT solution was removed and $100 \mu \mathrm{l}$ dimethyl sulfoxide (DMSO) was added to dissolve the colored formazan crystals for $15 \mathrm{~min}$. The absorbance at $490 \mathrm{~nm}$ of each aliquot was measured using a Sunrise RC micro plate reader (TECAN, Switzerland).

\section{Transfection and RNA interference}

We cloned hZnT7 into enhanced green fluorescence protein (EGFP) vector and sub-cloned hZnT7 or hZnT7-EGFP into pcDNA3.1/myc-hisA vector. The presence of the hZnT7 RNA in the cells was confirmed by RT-PCR. ZnT7 siRNA (Stealth RNAi) was prepared by Invitrogen (USA). The sequence of siRNA targeting a specific sequence in ZnT7 mRNA was 5'-GCCAUAGUCACGAAGCCAATT-3'. The scrambled siRNA sequence was 5'- UUGGCUUCGUGACUAUGGCTT-3'. The target sequences of ZnT7 siRNA and control ZnT7 siRNA were searched with BLAST in the GenBank database (accession ID: NM_133496). All transfection experiments were accomplished in independent triplicates in accordance with the manufacture's protocol for Lipofectamine 2000 and described previously (5). Cells were allowed to recover in DMEM for $24 \mathrm{~h}$ after transfection.

\section{Immunofluorescence analysis}

For single labeling with $\alpha$-SMA or E-cadherin, the cells were preincubated with normal donkey serum (NDS) (1:20) for $1 \mathrm{~h}$ and then incubated with mouse $\alpha$-SMA antibody (1:50) overnight. The sections were incubated with Texas Red-conjugated donkey anti-mouse IgG (1:50) for $2 \mathrm{~h}$ after several rinses. The cells were mounted with an anti-fading mounting medium and examined using a macro zoom fluorescence microscope (MVX10, Olympus, Japan). Images were taken using a microscope equipped with a digital color camera (DP71, Olympus, Japan). For double labeling, the cells were preincubated with NDS for $1 \mathrm{~h}$ and then incubated overnight in a monoclonal anti-trans-Golgi network (TGN38) antibody and ZnT7 (1:50). The cells were incubated for $2 \mathrm{~h}$ with a mixture of secondary antibodies, FITC-conjugated donkey anti-rabbit IgG (1:50, for labeling ZnT7) and Texas Red-conjugated donkey anti-mouse IgG (1:50, for labeling TGN38). The sections were mounted with an anti-fading mounting medium and examined using a confocal laser scanning microscope (SP2, Leica, Germany). Excitation filters for FITC (488 nm) and Texas-Red (568 nm) were used. Images were processed using an Adobe Photoshop program.

\section{Western Blot}

NRK-52E cells were lysed in RIPA buffer (ThermoScientific, Waltham, MA, USA). The protein concentration was determined by DC Protein Assays (BioRad, Hercules, CA, USA) in accordance with manufacturers' instructions. The used primary antibodies were summarized in Table 1. These separated proteins in SDS-PAGE were electrotransferred to Hybond-polyvinylidenefluoride (PVDF) membrane. The soaked PVDF membrane was then incubated in TBST containing primary antibodies overnight at $4^{\circ} \mathrm{C}$, 


\section{Kidney \\ Blood Pressure Research}

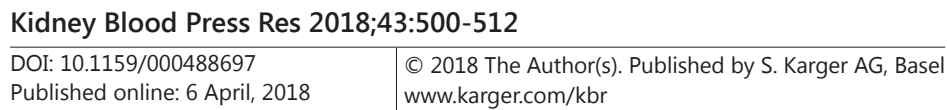

Zhang et al.: ZnT7 on Epithelial-to-Mesenchymal Transition in Renal Tubular Epithelial Cells

Table 1. The primary antibodies used in the experiments

\begin{tabular}{lccc}
\hline Antibody & ID number & Manufacturer & Concentration \\
\hline rabbit monoclonal anti-ZnT7 & \#SAB1303313 & Sigma-Aldrich, St. Louis, MO, USA & $1: 400$ \\
rabbit polyclonal anti-vimentin & \#SAB4503081 & Sigma-Aldrich, St. Louis, MO, USA & $1: 250$ \\
mouse monoclonal anti- $\alpha$-SMA & \#SAB5500002 & Sigma-Aldrich, St. Louis, MO, USA & $1: 400$ \\
mouse monoclonal anti- $\beta$-actin & \#BS6007MH & Bioworld Technology, Inc., St. Louis Park, MN, USA & Santa Cruz \\
rabbit monoclonal anti-E-cadherin & \#SC-71008 & Biotechnology, Inc. USA & $1: 400$ \\
rabbit polyclonal anti-phospho p38 & \#SAB450449 & Sigma-Aldrich, St. Louis, MO, USA & $1: 400$ \\
rabbit polyclonal anti-total JNK & \#SAB4200176 & Sigma-Aldrich, St. Louis, MO, USA & $1: 1000$ \\
rabbit polyclonal anti-phospho JNK & \#SAB4504449 & Sigma-Aldrich, St. Louis, MO, USA & $1: 1000$ \\
rabbit polyclonal anti-total ERK & \#SAB1305560 & Sigma-Aldrich, St. Louis, MO, USA & $1: 1000$ \\
rabbit polyclonal anti-phospho ERK1/2 & \#SAB1306604 & Sigma-Aldrich, St. Louis, MO, USA & $1: 800$ \\
rabbit monoclonal anti-phospho Smad2 & \#SAB4300251 & Sigma-Aldrich, St. Louis, MO, USA & $1: 800$ \\
rabbit monoclonal anti-phospho Smad3 & \#SAB4504210 & Sigma-Aldrich, St. Louis, MO, USA & $1: 800$ \\
\hline
\end{tabular}

washed with TBST buffer and incubated at room temperature for 2 hours in TBST containing horseradish peroxidase conjugated goat anti-mouse and goat anti-rabbit IgG Ab (Santa Cruz Inc, USA). The membranes were incubated in ECL (Pierce, Thermo Co. Ltd, USA) reagent for HRP (30s) and exposure to autoradiography film for visualization of the bands. The relative amounts of various proteins were analyzed. The results were quantified by Quantity One Software.

\section{Zinc analysis}

To analyze the distribution and concentration of zinc in the cells, N-(6-methoxy-8-quinolyl)-ptoluenesulfonamide (TSQ) fluorescence staining was performed as described previously [21]. TSQ is a one-step staining procedure and by the stoichiometric formation of zinc. Therefore, this method can be used for histochemically visualizing and quantitatively estimating the reactive zinc in tissues. The cultured NRK-52E cells were immersed in a solution of $4.5 \mu \mathrm{M}$ TSQ (Molecular Probes, Eugene, OR, USA) in $140 \mathrm{mM}$ sodium barbital and $140 \mathrm{mM}$ sodium acetate buffer ( $\mathrm{pH} \mathrm{10.5)} \mathrm{for} 1 \mathrm{~min}$. TSQ binding was imaged with a fluorescence microscope.

\section{Statistical Analysis}

Statistical analysis was performed using SPSS (Version 18, IBM Corporation, Armonk, NY, USA). Data were expressed as means \pm standard error (SEM). Variance was homogenous for use of standard ANOVA methodology. After statistical significance was established by ANOVA, individual comparisons were made using Tukey's multiple comparison test. The level of significance was defined at $\mathrm{P}<0.05$.

\section{Results}

\section{Intracellular $\mathrm{Zn}^{2+}$ levels in HG-stimulated NRK-52E cells}

We evaluated free intracellular $\mathrm{Zn}^{2+}$ levels in normal and HG-stimulated NRK-52E cells at concentration of $30 \mathrm{mM}$ for $12 \mathrm{~h}, 24 \mathrm{~h}, 48 \mathrm{~h}$, and $72 \mathrm{~h}$, respectively. TSQ fluorescence staining showed that the intracellular $\mathrm{Zn}^{2+}$ levels in HG-stimulated NRK-52E cells after $24 \mathrm{~h}$ were significantly decreased compared with non-stimulated NRK-52E cells (Fig. 2 a-f).

\section{ZnT7 expression levels in HG-stimulated NRK-52E cells}

We examined ZnT7 expression levels with Western blot under non-stimulated and HG conditions and demonstrated that ZnT7 expression was increased in HG-stimulated NRK52E cells after $48 \mathrm{~h}$ (Fig. $3 \mathrm{a}$ and b). To determine whether ZnT7 protected HG-induced EMT, we analyzed the effects of HG on EMT by modulating ZnT7 expression in the cells with both siRNA knock-down and over-expression techniques. ZnT7 expression at $48 \mathrm{~h}$ by using siRNA knock-down was reduced by approximately 65\% (Fig. 3c and d), while the highest expression level after transfection of hZnT7-EGFP was found at $48 \mathrm{~h}$ (Fig. 3e and f). 


\section{Kidney \\ Blood Pressure \\ Research}
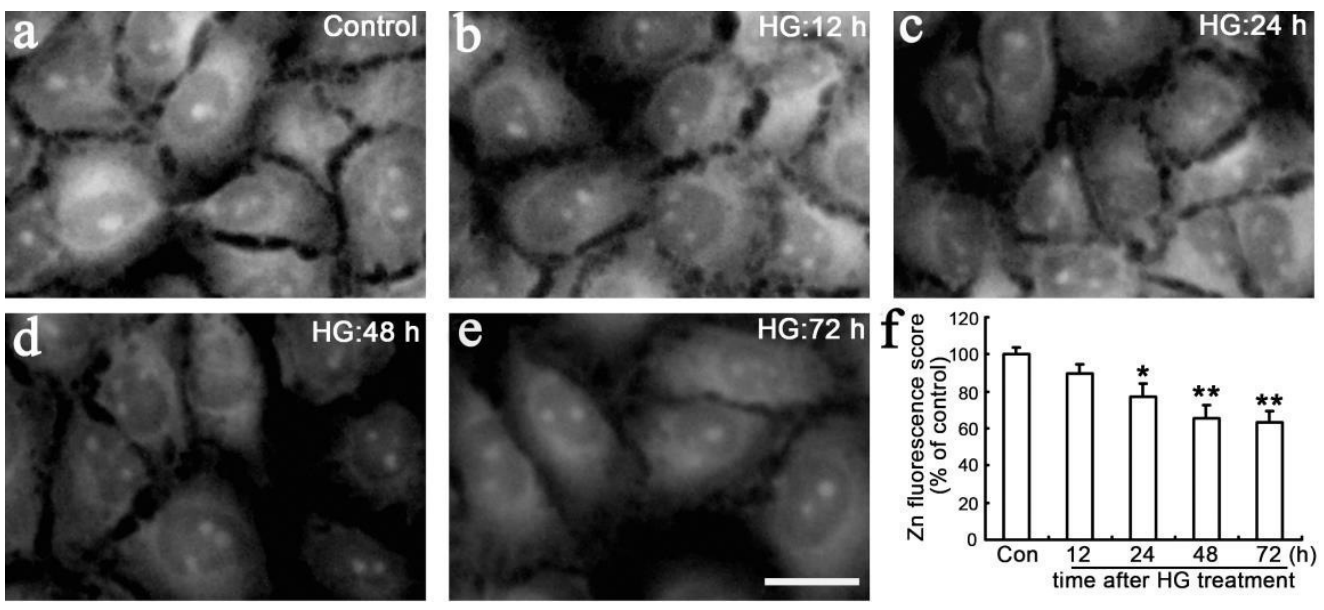

Fig. 2. Intracellular Zn2+ levels in HG-stimulated NRK-52E cells. Stimulation of high glucose (HG at $30 \mathrm{mM}$ ) in NRK-52E cells was performed for 12 h, 24 h, 48 h, and 72 h (n=10), respectively (Fig. 2a-e). Relative TSQ fluorescaping scores indicating free intracellular $\mathrm{Zn} 2+$ levels were analyzed $(n=10)$ and summarized in Fig. 2f. Scale bars $=10 \mu \mathrm{m} .{ }^{*} \mathrm{P}<0.05$ vs. control group; ${ }^{* *} \mathrm{P}<0.001$ vs. control group.

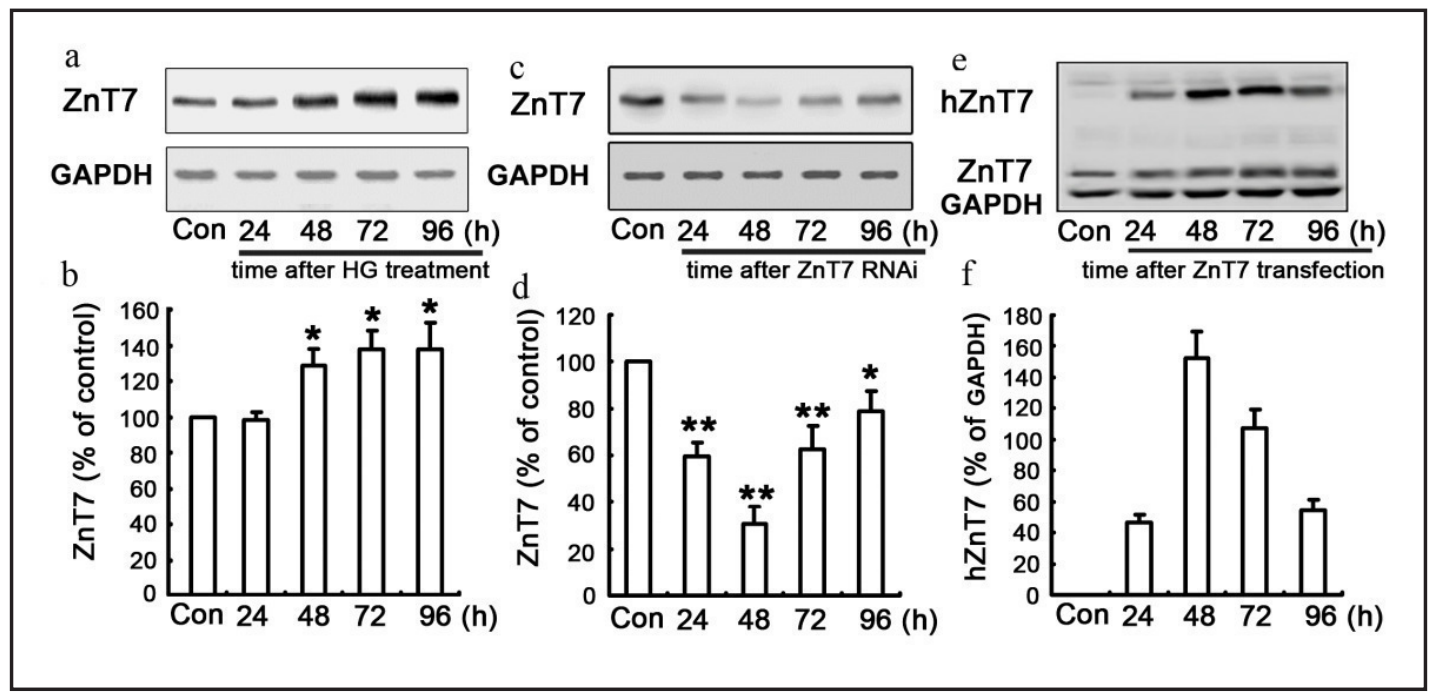

Fig. 3. ZnT7 expression levels in HG-stimulated NRK-52E cells. After NRK-52E cells were treated with 30 mM HG for $24 \mathrm{~h}, 48 \mathrm{~h}, 72 \mathrm{~h}$ and $96 \mathrm{~h}$, relative ZnT7 protein expression levels were assessed with Western blot. The amount of protein loaded is $20 \mu \mathrm{g}$ (Fig. 3a and b). ZnT7 expression was increased in HG-stimulated NRK-52E cells after $48 \mathrm{~h}$. However, ZnT7 expression at $48 \mathrm{~h}$ was decreased by using siRNA knock-down (Fig. 3c and d), while the highest ZnT7 expression was observed after transfection of hZnT7-EGFP (Fig. 3e and f). Each value represents the means \pm SEM $(n=10)$. The observed molecular weights of ZnT7-EGFP, hZnT7, ZnT7 and GAPDH were 85, 65, 42 and 36 Da, respectively. All experiments were performed at least three times. ${ }^{*} \mathrm{P}<0.05$ vs. control group; ${ }^{*} \mathrm{P}<0.001$ vs. control group.

\section{Dual immunofluorescence of ZnT7 and TGN38}

To observe the subcellular distribution of ZnT7 in the NRK-52E cells, double labeling of ZnT7 and TGN38 (the trans-Golgi marker) was used. ZnT7 was mainly localized in the perinuclear region, which was consistent with its Golgi apparatus localization (Fig. 4a). TGN38 was also found mainly residing in the perinuclear region of the cell (Fig. 4b). The 


\section{Kidney \\ Blood Pressure \\ Research}

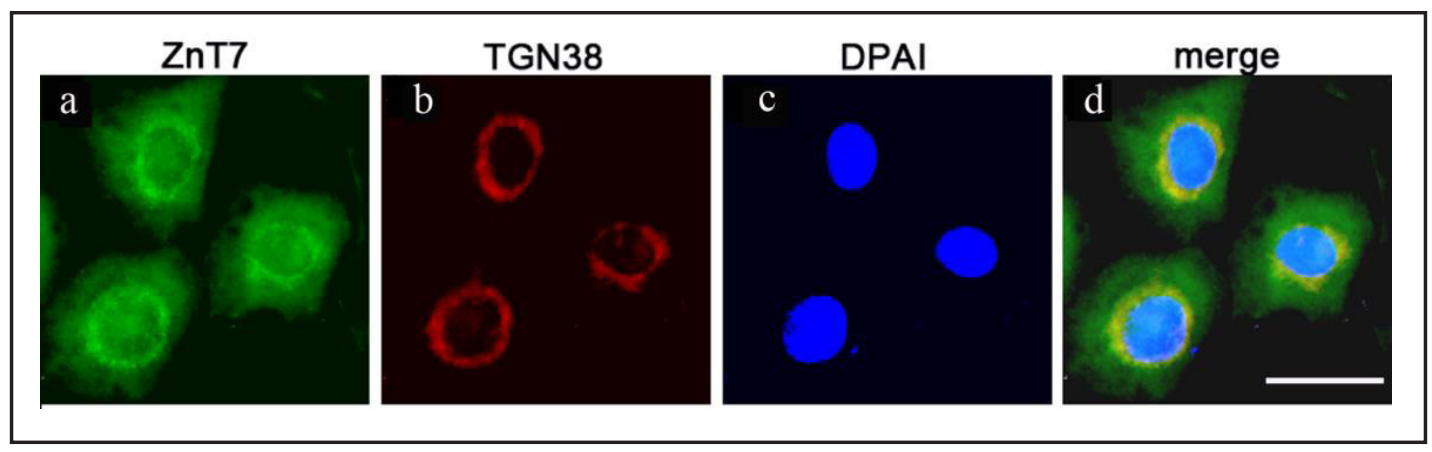

Fig. 4. Dual immunofluorescence of ZnT7 and TGN38. The intensity of ZnT7 staining was exclusively located in perinuclear region of the cells (Fig. 4a), while the intensity of TGN38 staining was located in perinuclear region of the cells (Fig. 4b). Fig. 4c shown the stained nucleus by DAPI. Merged images indicated a colocalization of ZnT7 and TGN38 (Fig. 4d). Scale bars $=10 \mu \mathrm{m}$.

nucleus was stained by DAPI and observed under a fluorescence microscope as described (Fig. 4c). After merging the ZnT7 and TGN38-labeled images, ZnT7 and TGN38 immunostaining overlapped in the in the perinuclear region of the cell (Fig. 4d). This result suggested that ZnT7 subcellular localization in Golgi apparatus.

\section{$\mathrm{ZnT7}$ expression levels and $\mathrm{Zn}^{2+}$ in the perinuclear region}

To evaluate the effect of ZnT7 on $\mathrm{Zn}^{2+}$ distribution, we employed TSQ fluorescence staining to visualize $\mathrm{Zn}^{2+}$ distribution in the perinuclear region of HG-stimulated NRK-52E cells. The images of $\mathrm{Zn}^{2+}$ distribution in the perinuclear region of non-HG-stimulated and HG-stimulated NRK-52E cells with either siRNA knock-down or over-expression of ZnT7 were represented in Fig. 5a-f, respectively. Zn fluorescence scores of each condition in the perinuclear region were summarized in Fig. 5g. A dispersed pattern of Zn staining was seen in the perinuclear region of the cells of HG, ZnT7 RNAi and HG/ZnT7 RNAi groups (Fig. 5b, d and f). Notably, a strong granular Zn staining was found in the perinuclear region of hZnT7 and HG/hZnT7 groups (Fig. 5c and e). The highest score of Zn fluorescence staining was detected in hZnT7 group. The fluorescent intensity in the HG/ZnT7 RNAi group was weaker than in any other group (Fig. $5 \mathrm{f}$ and g).

\section{Effect of ZnT7 on HG-induced EMT}

We further evaluate the effect of ZnT7 on HG-induced EMT of NRK-52E cells. After transfection of ZnT7 siRNA or hZnT-7-pcDNA3.1/myc-HisA, the NRK-52E cells in these two groups were treated with HG for $48 \mathrm{~h}$. First, the cell viability was studied by MTT analysis and no significant difference was detected between cells transfected with hZnT7-EGFP or ZnT7 siRNA. However, after treatment with HG for $48 \mathrm{~h}$, the cell viability in hZnT-7pcDNA3.1/myc-HisA/HG group was higher than that in the group with HG alone, while the ZnT7/siRNA/HG group was lower than that of HG group (See Fig. 1). Next, HG treatment in the NRK-52E cells for $48 \mathrm{~h}$ led to cell morphological changes to a fibroblast-like shape (Fig. 5 ). Meanwhile, $\alpha$-SMA and vimentin (the specific markers for myofibroblasts) were found to be up-regulated (Fig. 6a1-4 and b1-4), while E-cadherin (a $\mathrm{Ca}^{2+}$-dependent cell adhesion molecule that plays a vital role in the maintenance of renal epithelial polarity) was downregulated (Fig. 6c1-4). ZnT7siRNA-transfected cells showed a markedly increased expression of $\alpha$-SMA and vimentin during HG-induced EMT, compared with cells just treated with HG (Fig. 6a4, b4 and c4). Whereas this HG-induced EMT can be attenuated by over-expressing ZnT7 in NRK-52E cells (Fig. 6a3, b3 and c3). These results suggest that ZnT7 expression is associated with HG-induced EMT in a way that knock down of ZnT7 increases EMT event. In contrast, over-expressing ZnT7 in NRK-52E cells significantly decreased HG-induced EMT, as evidenced by the reduced up-regulation of $\alpha$-SMA and vimentin, and ameliorated expression of E-cadherin (Fig. 6d-g). 


\section{Kidney \\ Blood Pressure \\ Research}

Kidney Blood Press Res 2018;43:500-512

\begin{tabular}{l|l}
\hline DOI: 10.1159/000488697 & (C) 2018 The Author(s). Published by S. Karger AG, Basel
\end{tabular} Cells

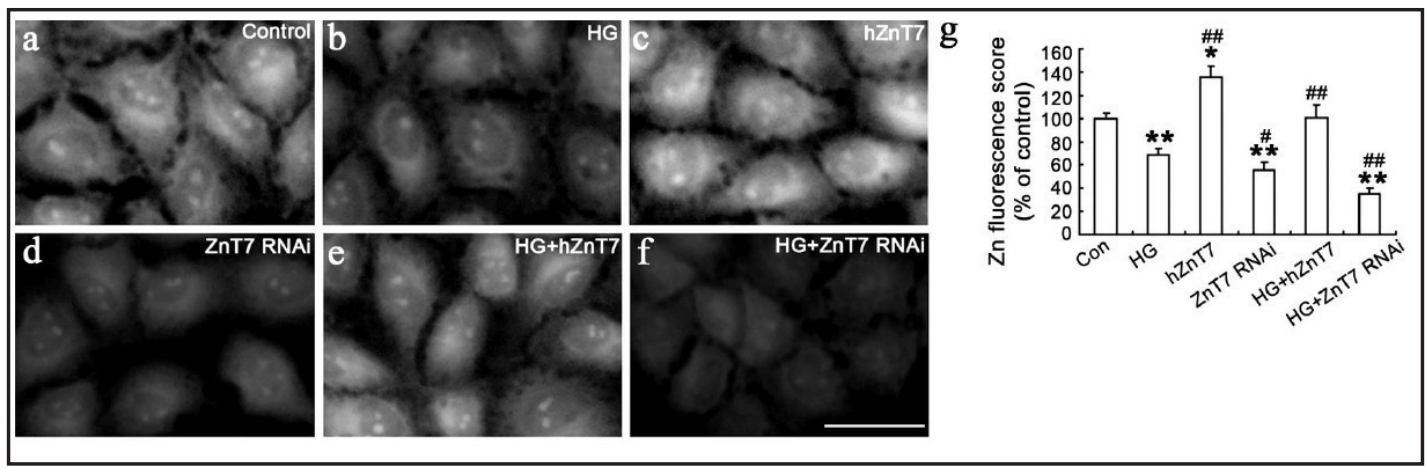

Fig. 5. ZnT7 expression levels and free Zn2+ distribution in NRK-52E cells. NRK-52E cells treated with HG, hZnT7, ZnT7 RNAi, HG/hZnT7 and HG/ZnT7 RNAi, respectively (Fig. 5b-f). Relative Zn fluorescing score was analyzed in each group $(\mathrm{n}=10)$ and summarized in Fig. 5 g. Scale bars $=20 \mu \mathrm{m}$. ${ }^{*} \mathrm{P}<0.05$ vs. control; ${ }^{* *} \mathrm{P}<0.001$ vs. control; \#\#P<0.001 vs. $\mathrm{HG}$ and $\# \mathrm{P}<0.05$ vs. HG.

Fig. 6. Effects of Transfection and RNA interference of ZnT7 on EMT and Effects of over-expression ZnT7 on expression of EMT markers. I $\mathrm{m} \mathrm{m} \mathrm{un} \mathrm{ofluore} \mathrm{s} \mathrm{cen} \mathrm{ce}$ analyses of EMT markers in NRK-52E cells, including $\alpha$-SMA (Fig. 6. a1-a4), vimentin (b1-b4) and E-cadherin (c1-c4). Cultures were untreated (a1-c1), exposed to HG (a2-c2), with $\mathrm{HG}+$ transfection of ZnT7 (a3-c3) or exposed to HG+transfection of ZnT7 siRNA (a4-c4). Results were representatives of two experiments. Magnification was $\times 200 \quad S$ c a l e bars $=20 \quad \mu \mathrm{m}$. NRK-52E cell treatments were then classified into six groups. Western blotting analyses of EMT marker expressions in NRK-52E cells. The corresponding protein levels were assessed using densitometry and expressed in relative intensities (Fig. 6d-g). Data were expressed as mean \pm SEM from at least three experiments. ${ }^{* *} \mathrm{P}<0.001$ vs. control; \#\#P<0.001 vs. HG; \#P<0.05 vs. HG.

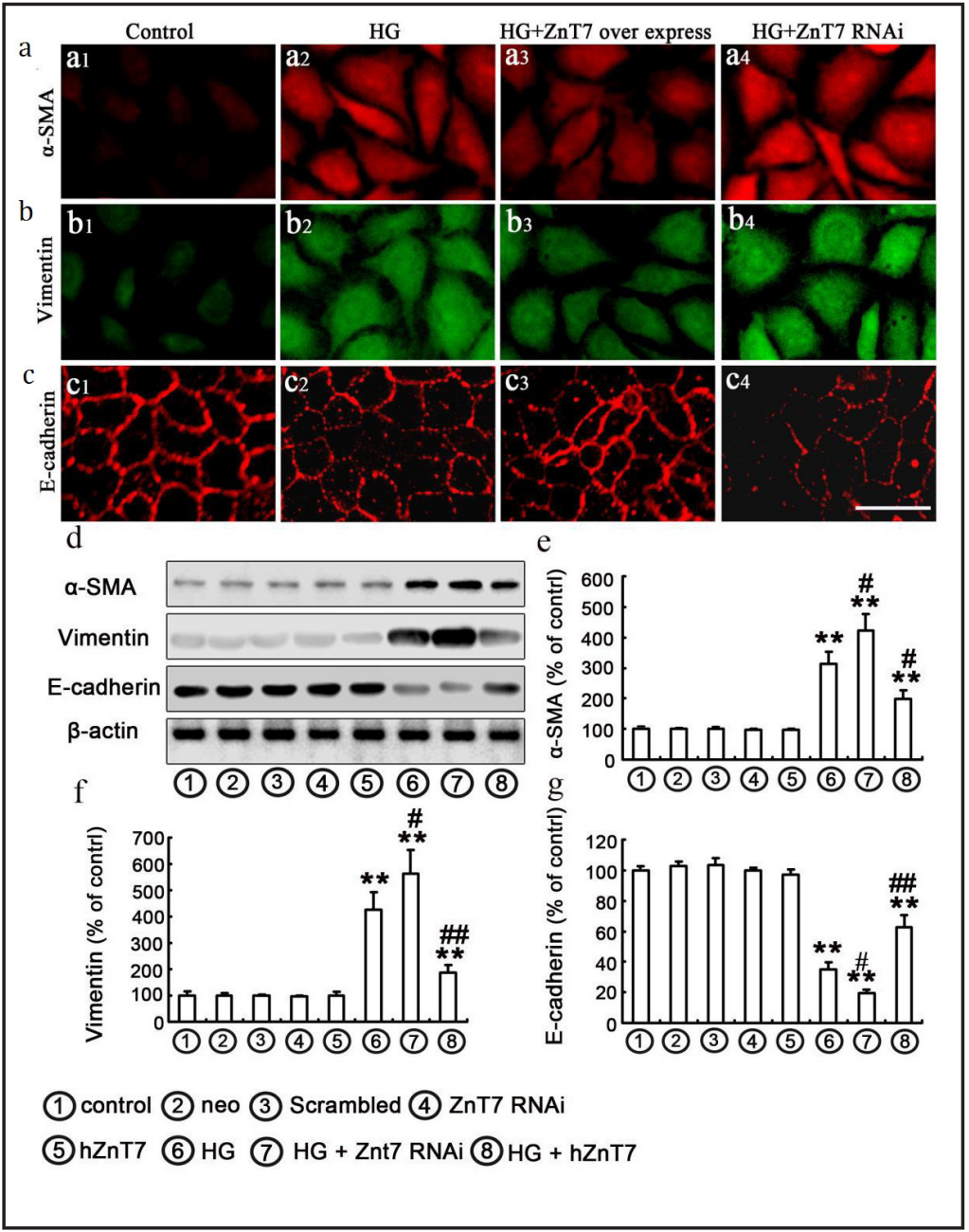

(1) control (2) neo (3) Scrambled (4) ZnT7 RNAi

(5)hZnT7 (6) HG (7) HG + Znt7 RNAi (8) HG + hZnT7 


\section{Kidney Blood Pressure Research}

Kidney Blood Press Res 2018;43:500-512

\begin{tabular}{l|l}
\hline DOI: $10.1159 / 000488697$ & (C) 2018 The Author(s). Published by S. Karger AG, Basel
\end{tabular}

Published onIIne: 6 April, 2018 www.karger.com/kb

Cells

\section{Effect of ZnT7 on the TGF- $\beta /$ Smad Pathway}

To understand whether ZnT7 mediated its effects on EMT in the NRK-52E cells through the TGF- $\beta /$ Smad pathway, we analyzed $p$-Smad2/3 levels under HG conditions by Western blot. Under the controlled conditions, ZnT7 over-expression or ZnT7 RNAi did not enhance p-Smad2/3 levels (Fig. 7a-d). The levels of p-Smad2/3 were significantly increased in HG group compared with the control group, which robust-enhanced in HG/ZnT7 RNAi group (Fig. 7a-d). Conversely, over-expression of ZnT7 significantly inhibited HG-induced levels of p-Smad2/3 (Fig. 7a-d). To confirm the involvement of TGF- $\beta /$ Smad signaling in the protective effect of ZnT7 on HG-induced EMT in NRK-52E cells, $10 \mu \mathrm{M}$ SB43152 (the inhibitor of TGF- $\beta$ receptor I) was added to the medium of NRK-52E cells for $1 \mathrm{~h}$ [22]. The cells were subsequently exposed to $\mathrm{HG}$ for $48 \mathrm{~h}$ after the treatment with ZnT7 RNAi. The expected results showed that HG or HG/ZnT7 RNAi group increased the expression of p-Smad2/3 and HG-induced EMT in the NRK-52E cells, i.e. the up-regulations of $\alpha$-SMA and vimentin were reduced, while the ameliorated expression of epithelial protein E-cadherin increased (Fig. 7e-j). However, the co-treatment with SB43152 effectively inhibited Smad2 and Smad3 phosphorylation and HG-induced EMT (Fig. 7e-j).

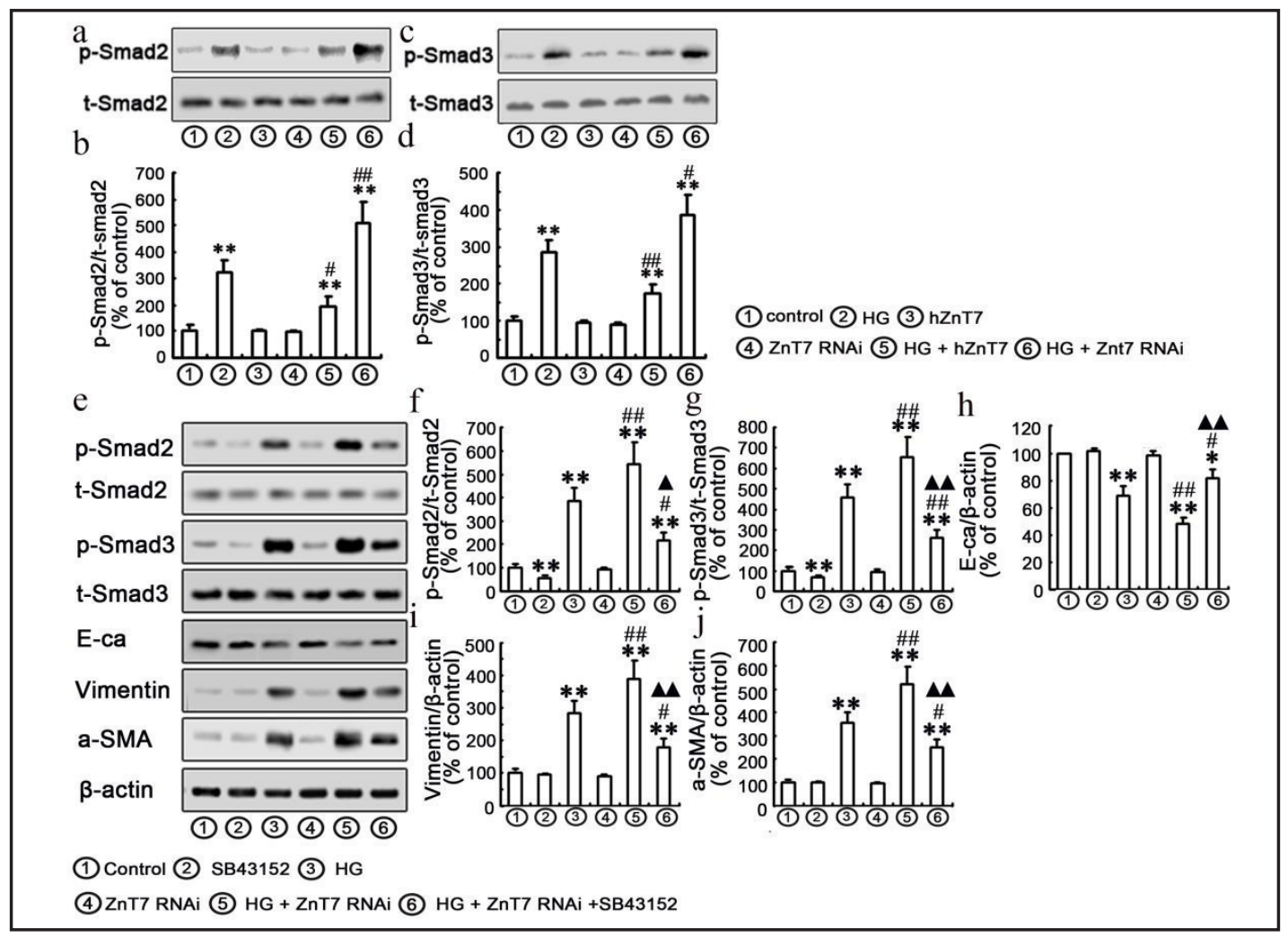

Fig. 7. Effect of ZnT7 on HG-induced TGF- $\beta$ /Smad signaling pathway in NRK-52E cells and its protection of HG-induced EMT. Relative p-Smad2/3 and t-Smad2/3 expression levels were calculated and normalized to the loading control with Western blotting analyses (Fig. 7a and c). The corresponding protein levels were then assessed using densitometry and expressed in relative intensities (Fig. $7 \mathrm{~b}$ and $\mathrm{d}$ ). All the results were obtained from three experiments. ${ }^{* *} \mathrm{P}<0.001$ vs. control; $\# \# \mathrm{P}<0.001, \# \mathrm{P}<0.05$ vs. HG. Furthermore, the cells were incubated with or without $10 \mu \mathrm{M}$ SB43152 for $1 \mathrm{~h}$ and subsequently exposed to HG for $48 \mathrm{~h}$ after ZnT7 RNAi for $24 \mathrm{~h}$. The protein expressions of EMT markers, t-Smad2/3 and p-Smad2/3 were analyzed with Western blot (Fig. 7e-j). Quantitative analysis was performed by measuring the fluorescence intensity compared with the control. Data were expressed as mean $\pm \mathrm{SEM}$ of at least three experiments. ${ }^{* *} \mathrm{P}<0.01$, vs. control, \#\# P<0.001, vs. HG, \# P<0.05, vs. HG, $\Delta \mathrm{P}<0.05$, vs. HG/ZnT7 RNAi, $\Delta \Delta \Delta \mathrm{P}<0.001$, vs. HG/ZnT7 RNAi. 


\section{Kidney Blood Pressure Research}

Kidney Blood Press Res 2018;43:500-512

\begin{tabular}{l|l}
\hline DOI: $10.1159 / 000488697$ & (C) 2018 The Author(s). Published by S. Karger AG, Basel
\end{tabular}

Published onlıne: 6 April, 2018 www.karger.com/kb

Cells

\section{Effect of ZnT7 on the MAPK Pathway}

The MAPK signaling pathway plays a role in EMT of the various kinds of epithelial cells and includes JNK, p38 MAPK and ERK [23-25]. We observed a robust increase of the phospho-p38, and phospho-ERK in the HG-treated NRK-52E cells compared with the control group (Fig. 8a and c). Conversely, over-expression ZnT7 significantly inhibited HG-induced phosphorylation of ERK (Fig. 8c and f) but no effect on phosphorylation of p38 MAPK was found (Fig. 8a and d). Moreover, there was no significant difference of phosphorylation of JNK in the HG, HG/ hZnT7 or HG/ZnT7 RNAi compared with the control group, respectively (Fig. $8 \mathrm{~b}$ and e). These results suggested that ZnT7 could be involved in HG-induced EMT through the regulation of the ERK pathway. Therefore, we further studied the ERK signaling pathway. The cells were incubated with or without $10 \mu \mathrm{M}$ of ERK inhibitor PD98059 for $1 \mathrm{~h}$ [23] and then exposed to HG for $24 \mathrm{~h}$ after ZnT7 RNAi. As expected, when compared with the cells treated with HG alone, the co-treatment of PD98059 with HG significantly decreased the expression of ERK phosphorylation (Fig. 9a and b). Once again, we found that ZnT7 RNAi significantly increased HG-induced EMT in NRK-52E cells However, pretreatment of these cells with PD98059 decreased the HG-induced EMT (Fig. 9a, c-e).

Fig. 8. Effect of ZnT7 on HG-induced MAPK signaling pathways. Relative p38, JNK, ERK, p-p38, p-JNK and p-ERK protein expressions were calculated with Western blot and normalized compared with the loading control (Fig. 8a-c). The corresponding protein levels were assessed using densitometry and expressed in relative intensities (Fig. 8d-f). Data were expressed as mean \pm SEM of at least three experiments. ${ }^{* *} \mathrm{P}<0.001$ vs. control; \#\# $\mathrm{P}<0.001, \# \mathrm{P}<0.05$ vs. HG.

Fig. 9. ZnT7 inhibits the ERK signaling pathway and protects the cells from HGinduced EMT. NRK-52E cells were pre-treated with or without $10 \quad \mu \mathrm{M}$ PD98059 for $1 \mathrm{~h}$ and subsequently exposed to HG for $48 \mathrm{~h}$ after ZnT7 RNAi for $24 \mathrm{~h}$. The protein expressions of EMT markers, t-ERK and p-ERK were assessed by Western blot analysis (Fig. 9a). Quantitative analysis was performed by measuring the

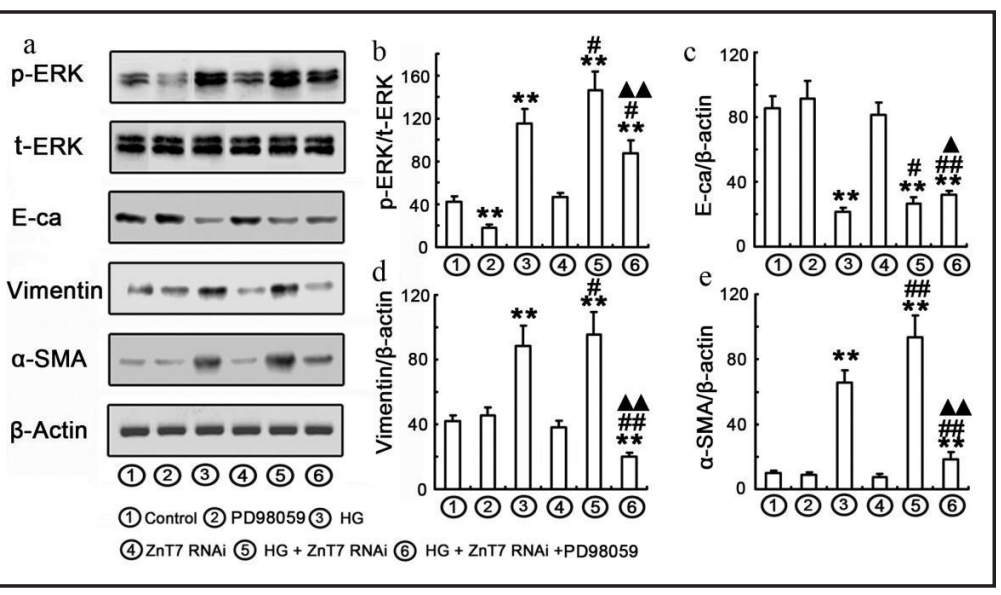
three experiments, ${ }^{* *} \mathrm{P}<0.001$ vs, control; \#\# $\mathrm{P}<0.001$ vs. $\mathrm{HG}$, \# $\mathrm{P}<0.05$ vs. $\mathrm{HG} ; \mathbf{A} \mathrm{P}<0.05$ vs. HG/ZnT7 RNAi; $\Delta \boldsymbol{\Delta} \mathrm{P}<0.001$ vs. HG/ZnT7 RNAi. 


\section{Kidney Blood Pressure Research}

Kidney Blood Press Res 2018;43:500-512

\begin{tabular}{l|l}
\hline DOI: 10.1159/000488697 & (C) 2018 The Author(s). Published by S. Karger AG, Base
\end{tabular}

Published onlıne: 6 April, 2018

www.karger.com/kbr

Zhang et al.: ZnT7 on Epithelial-to-Mesenchymal Transition in Renal Tubular Epithelial

Cells

\section{Discussion}

In the present study, we first observed that ZnT7 not only was localized in the perinuclear region and Golgi apparatus but also up-regulated in HG-induced EMT of NRK-52E cells. We then found that ZnT7 had a protective effect on HG-induced EMT. Furthermore, we explored the cellular mechanism of ZnT7 in the MAPK/ERK and TGF- $\beta$ /Smad pathways.

ZnT7 is localized on the membrane of the Golgi/endoplasmic reticulum (ER) and vesicular compartments [3-5]. The function of ZnT7 is to facilitate zinc transport from the cytoplasm into the Golgi apparatus and to regulate cellular zinc homeostasis [3]. In the present study, we used dual immunofluorescence of ZnT7 together with TGN38 and observed that ZnT7 was mainly located in the perinuclear region and Golgi apparatus of the NRK-52E cell, which is consistent with the reports from our $[9,10]$ and other groups $[11,26]$. Furthermore, we found that ZnT7 facilitated Zn transport from the cytoplasm into the Golgi apparatus in the cells within $48 \mathrm{~h}$ of HG treatment. Over-expressing ZnT7 in NRK-52E cells significantly decreased HG-induced EMT, as evidenced by the reduced up-regulation of $\alpha$-SMA, vimentin, and the ameliorated expression of epithelial protein E-cadherin. The levels of free zinc ions in the NRK-52E cells were significantly increased after over-expression of ZnT7.

We have previously reported that ZnT7 inhibited HG-induced EMT and apoptosis in RPMC $[9,10]$, and further designed the present study to understand whether ZnT7 has the similar effect but in NRK-52E cells. A previous study showed that over-expression of ZnT7 in Chinese hamster ovarian cells under high zinc conditions lead to accumulation of free $\mathrm{Zn}^{2+}$ in the Golgi apparatus [3]. A recent study demonstrated that hyperglycemia decreased ZnT7 activity following redistribution of cellular free $\mathrm{Zn}^{2+}$, which plays an important role during ER stress in cardiomyocytes [11]. In our present study, HG increased ZnT7 expression and over-expression of ZnT7 induced accumulation of free $\mathrm{Zn}^{2+}$ in perinuclear region and Golgi apparatus of NRK-52E cells. These results implicated that ZnT7 transport zinc into Golgi apparatus in the HG-induced EMT in NRK-52E cells, which was consistent with our previous studies in RPMCs $[9,10]$. The up-regulation of ZnT7 in NRK-52E cells suggested that ZnT7 was involved in mobilizing Zn from the cytoplasm into the Golgi apparatus and subsequently indicated a need to reduce $\mathrm{Zn}$ efflux from the Golgi apparatus and to maintain Zn levels during HG conditions. Another previous study demonstrated that the Golgi apparatus can influence the expression of EMT-maker proteins [27]. Considering the role of Golgi apparatus is for protein synthesis and post-translational modification, we thereby considered that ZnT7 may protect NRK-52E cells form HG-induced EMT by regulating EMT gene transcription. These results from the present study thus supported our hypothesis that ZnT7 has a protective effect on EMT of NRK-52E cells. Considering that the gene therapy for ZnT7 is not an available resource yet, Zn supplementation to diabetic patients may induce ZnT7 expression. Further investigation with transgenic animal model to induce a therapeutic increase of ZnT7 expression in vivo has been taken into our consideration.

To understand the possible mechanism of the protection of ZnT7 on EMT, we analyzed the key molecules of the TGF- $\beta$ /Smad and MAPK/ERK pathways by using hZnT7-EGFP and ZnT7 siRNA in NRK-52E cells. Previous studies indicated that TGF- $\beta 1$ as a stronger stimulator of EMT. Inhibition of TGF- $\beta /$ Smad signaling has been considered a central mechanism to prevent earlier renal fibrosis [17]. TGF- $\beta$ receptor I phosphorylates Smad2 and 3 and subsequently associates with the common partner Smad 4, which translocate into the nucleus and activate the expression of TGF- $\beta$-responsive genes [28, 29]. SzusterCiesielska et al. previously reported that $\mathrm{Zn}$ supplementation decreases ethanol- and acetaldehyde-induced activation of liver stellate cells and inhibit liver fibrosis changes though inhibiting TGF- $\beta /$ Smad signaling [30]. Maywald et al. have very recently demonstrated that zinc supplementation increased TGF- $\beta 1$-dependent regulatory $\mathrm{T}$ cell induction in allogeneic reaction due to a triggered intracellular zinc signal, which in association with an increased Smad 2/3 activation [31]. Data from the present study suggested that TGF- $\beta$ / 


\section{Kidney \\ Blood Pressure Research}

Kidney Blood Press Res 2018;43:500-512

\begin{tabular}{l|l}
\hline DOI: $10.1159 / 000488697$ & (C) 2018 The Author(s). Published by S. Karger AG, Basel
\end{tabular}

Published onlıne: 6 April, 2018

www.karger.com/kb

Smad signaling activation might also occur through this process in NRK-52E cells with HG treatment, and also implicated that ZnT7 protect HG-induced EMT in NRK-52E cells partly by inhibiting TGF- $\beta /$ Smad signaling.

The MAPK pathway is a highly conserved pathway involved in diverse cellular functions, including cell proliferation, cell differentiation and apoptosis, while JNK, p38 MAPK and ERK in this pathway are involved in a $\mathrm{Zn}$-sensing process $[32,33]$. The results from the present study indicated that over-expression ZnT7 significantly inhibited HG-induced expression of phospho-ERK but not phospho-p38 MAPK. There was no difference of the expression of phospho-JNK in the HG, HG/hZnT7 or HG/ZnT7 RNAi groups. A previous study showed that MAPK signaling from the Golgi apparatus, in striking contrast to the plasma membrane, generated a graded ERK output [34]. Data from our experiments confirmed that ZnT7RNAi under HG conditions triggered a rapid activation of ERK, which then initiated downstream signaling events including enhanced HG-induced EMT. Moreover, chemical inhibition of ERK with PD98059 completely suppressed HG-stimulated EMT and increased cellular injury. Therefore, we interpreted that endogenous ZnT7 protected HG-induced EMT in NRK-52E cells through inhibiting not only the activation of TGF- $\beta /$ Smad but also ERK signaling pathways.

There is growing evidence suggesting that tubulointerstitial fibrosis processes play an important role in the pathogenesis of DN $[13,14]$. EMT in renal tubular epithelial cells, as a phenotypic conversion, plays a major role in renal interstitial fibrogenesis under pathologic condition [35]. Recent studies indicated that hyperglycemia could induce EMT of renal tubular epithelial cells, acting as a critical mechanism of renal tubulointerstitial fibrosis in DN $[15,16]$. EMT course of tubular epithelial cells consists of four key steps: (1) loss of epithelial cell adhesion; (2) de novo a-SMA expression and actin reorganization; (3) disruption of the tubular basement membrane; and (4) enhanced cell migration and invasion [17]. Although EMT is not the only process involved in fibrosis, data from the present and other studies suggest that blocking EMT may prevent, attenuate or delay fibrosis.

\section{Conclusion}

The present study provides the first evidence that ZnT7 has a protective effect on HGinduced EMT in NRK-52E cells and suggests that the activity of ZnT7 in HG-induced EMT of the NRK-52E cells is most likely involved in the MAPK/ERK and TGF- $\beta /$ Smad pathways. Considering that EMT plays an important role in the development and progression of interstitial fibrosis in DN, ZnT7 may be a potential target for therapeutic intervention research of DN. Further investigation of ZnT7 in the pathogenesis of DN has been taken into our consideration.

\section{Abbreviations}

AAS (atomic absorption spectrophotometry); DCF-DA (2, 7-dichlorofluoresceindiacetate); DMEM (Dulbecco's modified Eagle's medium); EMT (Epithelial-to-mesenchymal transition); EGFP (enhanced green fluorescence protein); FITC (fluorescein isothiocyanate); NDS (normal donkey serum); NRK-52E (cells, normal rat kidney tubular epithelial cells); RTEC (renal tubular epithelial cells); PBS (phosphate-buffered saline); PI (propidium iodide); RIPA (radioimmune precipitation assay); SMA (Smooth muscle cell actin); TBS (trisbuffered saline); TGF (transforming growth factor); TGN (trans-Golgi network); ZnT7 (zinc transporter 7). 


\section{Kidney \\ Blood Pressure Research}

Kidney Blood Press Res 2018;43:500-512

\begin{tabular}{l|l}
\hline DOI: 10.1159/000488697 & (c) 2018 The Author(s). Published by S. Karger AG, Basel \\
\hline
\end{tabular}

Published onlıne: 6 April, 2018

Zhang et al.: ZnT7 on Epithelial-to-Mesenchymal Transition in Renal Tubular Epithelial Cells

\section{Disclosure Statement}

There was no potential conflict of interest relevant to this article.

\section{Acknowledgements}

This work was supported by the Natural Science Foundation of China (81670670, 81170561), and Postdoctoral Science Foundation of China (2014MM551144), and the Liaoning Province Science and Technology Plan Project (nos. 201602405).

\section{References}

1 Vallee BL, Falchuk KH: The biochemical basis of zinc physiology. Physiol Rev 1993;73:79-118.

2 Walsh CT, Sandstead HH, Prasad AS, Newberne PM, Fraker PJ: Zinc: health effects and research priorities for the 1990s. Environ Health Perspect 1994;102:5-46.

3 Kirschke CP, Huang L: ZnT7, a novel mammalian zinc transporter, accumulates zinc in the Golgi apparatus. J Biol Chem 2003;278:4096-4102.

-4 Huang L, Yu YY, Kirschke CP, Gertz ER, Lloyd KKC: Znt7 (Slc30a7)-deficient mice display reduced body zinc status and body fat accumulation. J Biol Chem 2007;282:37053-37063.

5 Liang D, Xiang L, Yang M, Zhang X, Guo B, Chen Y, Yang L, Cao J: ZnT7 can protect MC3T3-E1 cells from oxidative stress-induced apoptosis via PI3K/Akt and MAPK/ERK signaling pathways. Cell Signal 2013;25:1126-1135.

6 Chen L, Ge ZJ, Wang ZB, Sun T, Ouyang YC, Sun QY, Sun YP: TGN38 is required for the metaphase I/ anaphase I transition and asymmetric cell division during mouse oocyte meiotic maturation. Cell Cycle 2014;13:2723-2732.

7 Huang L, Yan M, Kirschke CP: Over-expression of ZnT7 increases insulin synthesis and secretion in pancreatic beta-cells by promoting insulin gene transcription. Exp Cell Res 2010;316:2630-2643.

8 Huang L, Kirschke CP, Lay YA, Levy LB, Lamirande DE, Zhang PH: Znt7-null mice are more susceptible to diet-induced glucose intolerance and insulin resistance. J Biol Chem 2012;287:33883-33896.

-9 Zhang X, Liang D, Guo B, Sun L, Chi ZH, Cai Y, Wang L, Ma J: Zinc transporter 7 induced by high glucose attenuates epithelial-to-mesenchymal transition of peritoneal mesothelial cells. Biol Trace Elem Res 2013;151:138-147.

10 Zhang X, Liang D, Guo B, Deng W,Chi ZH, Cai Y, Wang L, Ma J: Zinc transporter 5 and zinc transporter 7 induced by high glucose protects peritoneal mesothelial cells from undergoing apoptosis. Cell Signal 2013;25:999-1010.

-11 Tuncay E, Bitirim VC, Durak A, Carrat GRJ, Taylor KM, Rutter GA, Turan B: Hyperglycemia-Induced Changes in ZIP7 and ZnT7 Expression Cause Zn2+ Release From the Sarco(endo)plasmic Reticulum and Mediate ER Stress in the Heart. Diabetes 2017;66:1346-1358.

$\checkmark 12$ Zimmet PZ, Magliano DJ, Herman WH, Shaw JE: Diabetes: a 21st century challenge. Lancet Diabetes Endocrinol 2014;2:56-64.

13 Yeh CH, Chang CK, Cheng KC, Li YX, Zhang YW, Cheng JT: Role of Bone Morphogenetic Proteins-7 (BMP7) in the Renal Improvement Effect of DangGui (Angelica sinensis) in Type-1 Diabetic Rats. Evid Based Complement Alternat Med 2011;2011:796723.

14 Gilbert RE, Cooper ME: The tubulointerstitium in progressive diabetic kidney disease: more than an aftermath of glomerular injury? Kidney Int 1999;56:1627-1637.

15 Simonson MS: Phenotypic transitions and fibrosis in diabetic nephropathy. Kidney Int 2007;71:846-854.

16 Burns WC, Twigg SM, Forbes JM, Pete J, Tikellis C, Thallas-Bonke V, Thomas MC, Cooper ME, Kantharidis P: Connective tissue growth factor plays an important role in advanced glycation end product-induced tubular epithelial-to-mesenchymal transition: implications for diabetic renal disease. J Am Soc Nephrol 2006;17:2484-2494. 


\section{Kidney \\ Blood Pressure Research}

Zhang et al.: ZnT7 on Epithelial-to-Mesenchymal Transition in Renal Tubular Epithelial Cells

17 Hills CE, Squires PE: The role of TGF-beta and epithelial-to mesenchymal transition in diabetic nephropathy. Cytokine Growth Factor Rev 2011;22:131-139.

18 Yang J, Liu Y: Dissection of key events in tubular epithelial to myofibroblast transition and its implications in renal interstitial fibrosis. Am J Pathol 2011;159:1465-1475.

19 Sun YB, Qu X, Caruana G, Li J: The origin of renal fibroblasts/myofibroblasts and the signals that trigger fibrosis. Differentiation 2016;92:102-107.

-20 Zeisberg M, Kalluri R: The role of epithelial-to-mesenchymal transition in renal fibrosis. J Mol Med (Berl) 2004;82:175-181.

-21 Frederickson CJ, Kasarskis EJ, Ringo D, Frederickson RE: A quinoline fluorescence method for visualizing and assaying the histochemically reactive zinc (bouton zinc) in the brain. J Neurosci Methods 1987;20:91103.

-22 Stewart A, Guan H, Yang K: BMP-3 promotes mesenchymal stem cell proliferation through the TGF-beta/ activin signaling pathway. J Cell Physiol 2010;223:658-666.

23 Zhang X, Wang J, Fan Y, Yang L, Wang L, Ma J: Zinc Supplementation Attenuates High Glucose-Induced Epithelial-to-Mesenchymal Transition of Peritoneal Mesothelial Cells. Biol Trace Elem Res 2012;150:229235.

-24 Yang HW, Lee SA, Shin JM, Park IH, Lee HM: Glucocorticoids ameliorate TGF- $\beta 1$-mediated epithelialtomesenchymal transition of airway epithelium through MAPK and Snail/Slug signaling pathways. Scientific Reports 2017;7:3486.

25 Sheng W, Chen C, Dong M, Wang G, Zhou J, Song H, Li Y, Zhang J, Ding S: Calreticulin promotes EGFinduced EMT in pancreatic cancer cells via Integrin/EGFR-ERK/MAPK signaling pathway. Cell Death Dis 2017;8:e3147.

26 Suzuki T, Ishihara K, Migaki H, Matsuura W, Kohda A, Okumura K, Nagao M, Yamaguchi-Iwai Y, Kambe T: Zinc transporters, ZnT5 and ZnT7, are required for the activation of alkaline phosphatases, zinc-requiring enzymes that are glycosylphosphatidylinositol-anchored to the cytoplasmic membrane. J Biol Chem 2005;280:637-643.

27 Jiang Y, Xie X, Li Z, Wang Z, Zhang Y, Ling ZQ, Pan Y, Wang Z, Chen Y: Functional cooperation of RKTG with p53 in tumorigenesis and epithelial- mesenchymal transition. Cancer Res 2011;71:2959-2968.

28 Shi Y, Massague J: Mechanisms of TGF-beta signaling from cell membrane to the nucleus. Cell 2003;113:685-700.

-29 Carvajal G, Rodríguez-Vita J, Rodrigues-Díez R, Sánchez-López E, Rupérez M, Cartier C, Esteban V, Ortiz A, Egido J, Mezzano SA, Ruiz-Ortega M: Angiotensin II activates the Smad pathway during epithelial mesenchymal transdifferentiation. Kidney Int 2008;74:585-595.

-30 Szuster-Ciesielska A, Plewka K, Daniluk J, Kandefer-Szerszen M: Zinc supplementation attenuates ethanol- and acetaldehydeinduced liver stellate cell activation by inhibiting reactive oxygen species (ROS) production and by influencing intracellular signaling. Biochem Pharmacol 2009;78:301-314.

-31 Maywald M, Meurer SK, Weiskirchen R, Rink L: Zinc supplementation augments TGF- $\beta 1$-dependent regulatory T cell induction. Mol Nutr Food Res 2017, DOI: 10.1002/mnfr.201600493.

-32 Liu Q, Mao H, Nie J, Chen W, Yang Q, Dong X, Yu X: Transforming growth factor \{beta\}1 induces epithelialmesenchymal transition by activating the JNK-Smad3 pathway in rat peritoneal mesothelial cells. Perit Dial Int 2008;28:S88-95.

33 Zhou Q, Zeng R, Xu C, Liu L, Chen L, Kou P, Pei G, Bai S, Zhang Y, Li C, Rong S, Han M, Xu G: Erbin inhibits TGF-beta1-induced EMT in renal tubular epithelial cells through an ERK-dependent pathway. J Mol Med (Berl) 2012;90:563-574.

-34 Inder K, Harding A, Plowman SJ, Philips MR, Parton RG, Hancock JF: Activation of the MAPK module from different spatial locations generates distinct system outputs. Mol Biol Cell 2008;19:4776-4784.

-35 Kriz W, Kaissling B, Le Hir M: Epithelial-mesenchymal transition (EMT) in kidney fibrosis: fact or fantasy? J Clin Invest 2011;121:468-474. 